Nutr Bras 2019;18(3):134-40

https://doi.org/10.33233/nb.v18i3.2837

\title{
ARTIGO ORIGINAL \\ Avaliação do estado nutricional e anemia ferropriva em crianças de 3 a 10 anos atendidas em uma unidade básica de saúde no município de Portel/PA \\ Assessment of nutritional status and iron deficiency anemia in 3 to 10 years old children at a basic health unit of Portel/PA
}

Rozinéia de Nazaré Alberto Miranda, D.Sc. ${ }^{*}$, Camila Corrêa Bandeira**, Patricia Rodrigues Portugal $^{\star \star *}$, Bruno Rafael Batista Ataide ${ }^{\star \star * *}$

*Docente da Faculdade de Nutrição, Instituto de Ciências da Saúde, Universidade Federal do Pará, **Bacharel em Nutrição, UFPA, ${ }^{* * *}$ Mestranda em Oncologia e Ciências Médicas - UFPA, ${ }^{* * \star * E s p e c i a l i s t a ~ e m ~ A t e n c ̧ a ̃ o ~ a ̀ ~ S a u ́ d e ~ M e n t a l, ~ U n i v e r s i d a d e ~ E s t a d u a l ~ d o ~ P a r a ́ ~}$

Recebido 19 de abril de 2019; aceito 10 de setembro de 2019.

Correspondência: Rozinéia de Nazaré Alberto Miranda, UFPA, Faculdade de Nutrição, Cidade Universitária Prof. José da Silveira Neto, Campus Profissional III, Complexo Saúde, Rua Augusto Corrêa, 01 Guamá Belém PA

Rozinéia de Nazaré Alberto Miranda: rozi@ufpa.br Camila Corrêa Bandeira: camilacb1020@gmail.com

Patricia Rodrigues Portugal: patriciarodriguesportugal@gmail.com

Bruno Rafael Batista Ataide: brunoataide8@hotmail.com

\section{Resumo}

A anemia ferropriva é uma das carências nutricionais predominantes ao redor do mundo e a repercussão a curto e longo prazo de uma alimentação inadequada nas crianças tem favorecido um estado nutricional inadequado. Objetivou-se conhecer o estado nutricional e a presença de anemia ferropriva em crianças de 3 a 10 anos atendidas em uma Unidade Básica de Saúde no município de Portel/Pará. Estudo descritivo, transversal, realizado a partir da aplicação de um questionário para obtenção de dados antropométricos e hematológicos. $O$ estudo revelou que $92,9 \%$ das crianças apresentaram-se eutróficas para o índice Peso para Idade (P/I) e 89,4\% para Estatura para idade (E/I). Quanto ao IMC/I 82,4\% apresentaram eutrofia e 12,4\% risco de sobrepeso, sem diferença significativa estatística entre os sexos. Nas variáveis hematológicas, constatou-se $42,9 \%$ das crianças apresentaram hemoglobina abaixo dos valores de normalidade. No teste de correlação de Pearson entre hemoglobina e hematócrito com os índices antropométricos apenas a hemoglobina se correlacionou significativamente exceto para o índice E/I. Analisando VCM, HCM e CHCM das crianças com hemoglobina baixa 84,9\% destas crianças apresentaram anemia ferropriva. A pesquisa mostrou prevalência de anemia ferropriva em crianças, evidenciando a importância do acompanhamento nutricional e alimentar nos primeiros anos de vida, como forma de prevenção de deficiências nutricionais subclínicas.

Palavras-chave: anemia ferropriva, estado nutricional, antropometria.

\begin{abstract}
Iron deficiency anemia is one of the most prevalent nutritional deficiencies around the world and the short and long-term repercussions of inadequate nutrition in children have favored an inadequate nutritional status. The aim of this study was to determine the nutritional status and the presence of iron deficiency anemia in children aged 3 to 10 years attending a Basic Health Unit in the city of Portel/Pará. This descriptive and cross-sectional study was performed through the application of a questionnaire to obtain anthropometric and hematological data. The study revealed that $92.9 \%$ of the children were eutrophic for the W/A index and $89.4 \%$ for the H/A. Regarding BMI/A, $82.4 \%$ presented eutrophy and $12.4 \%$ risk of overweight, with no statistically significant difference between the sexes. In hematological variables, $42.9 \%$ of the children had hemoglobin below normal values. In the Pearson correlation test between hemoglobin and hematocrit with the anthropometric indices only hemoglobin correlated significantly except for the
\end{abstract}


$\mathrm{H} / \mathrm{A}$ index. Analyzing VCM, HCM and CHCM of children with low hemoglobin, $84.9 \%$ of these children presented iron deficiency anemia. The study showed the prevalence of iron deficiency anemia in children, evidencing the importance of nutritional and food monitoring in the first years of life, as a way of preventing subclinical nutritional deficiencies.

Key-words: iron deficiency anemia, nutritional status, anthropometry

\section{Introdução}

A alimentação infantil é um assunto que nos últimos anos tem estimulado um grande interesse em várias áreas do conhecimento, visto que envolve diversos aspectos além dos nutricionais. As repercussões a curto e longo prazo de uma alimentação inadequada tem favorecido a busca por um melhor entendimento de como o hábito alimentar é construído e assim, incitar a atenção das pessoas para a enorme relevância dos hábitos alimentares nos primeiros anos de vida [1].

As crianças se caracterizam como um grupo de grande fragilidade devido ao crescimento acelerado e à imaturidade imunológica e fisiológica. A nutrição apropriada nos primeiros anos de vida é a base para o crescimento e desenvolvimento saudáveis. O consumo inadequado de nutrientes pode prejudicar o estado nutricional e acarretar carências ou excessos nutricionais. As doenças carências ampliam a predisposição das crianças a infecções, além de poder comprometer o amadurecimento do sistema nervoso, visual, mental e intelectual. No Brasil, as deficiências de ferro e vitamina $A$ são as carências que mais se destacam e configuram um problema de saúde pública [2].

A anemia ferropriva é uma das carências nutricionais predominantes ao redor do mundo, pode ocorrer devido à perda sanguínea crônica, ingestão ou absorção de ferro reduzida, perdas urinárias e aumento do volume sanguíneo. $\mathrm{Na}$ anemia por deficiência de ferro (ADF) ocorre diminuição da quantidade de ferro no plasma sanguíneo. Há uma depleção no local de armazenamento de ferro dentro dos macrófagos, portanto, estes não podem provê-lo para 0 plasma. Em consequência disso, a concentração plasmática do mineral reduz a níveis que restringem a eritropoese. Os grupos atingidos com maior frequência são crianças menores de 5 anos, lactentes e mulheres em idade fértil [3].

Estudos epidemiológicos estimam que $20 \%$ a $25 \%$ das crianças no mundo possuem anemia por deficiência de ferro. No presente momento, constam-se que o avanço da doença pode estar associado a alguns fatores socioeconômicos como a baixa renda da família da criança, muitas pessoas compartilhando a mesma casa e a baixa escolaridade dos pais [4]. A ocorrência de anemia advém de múltiplos fatores, sendo eles, biológicos, ambientais, socioeconômicos, de origem alimentar e nutricional, assim como de problemas de saúde. Contudo, nota-se que a prevalência da ADF no período da infância está relacionada com o fato da criança necessitar de altas quantidades de ferro para auxiliar em seu crescimento e desenvolvimento acelerados e não atingir essas necessidades devido a dietas pobres em ferro heme [5].

O diagnóstico da anemia pode ser feito através de exames clínicos e laboratoriais. Exames como hemograma, saturação de transferrina e ferritina sérica, verificação dos níveis de piridoxina, vitamina B12 e ácido fólico, além de investigações de perdas sanguíneas, assim como doenças de base como lúpus e anemia falciforme podem ser indícios da presença de anemia [6].

Quando o diagnóstico de deficiência de ferro e anemia é confirmado, uma das orientações é ampliar a ingestão de ferro através da alimentação, já que é uma opção de baixo custo e não acarreta efeitos colaterais. Por meio da dieta, são ofertados dois tipos de ferro: o ferro heme ou orgânico que é originário de fontes animais e o ferro não heme ou inorgânico que provém de alimentos de origem vegetal. Entretanto, o ferro de origem animal é absorvido em maior quantidade se comparado ao de origem vegetal [7].

O aleitamento materno exclusivo fornece um baixo conteúdo de ferro, contudo, a quantidade ofertada, supre todas as necessidades do lactente que nasceu a termo, sendo suficiente durante os primeiros seis meses de vida. Passado esse período, estudos constatam uma baixa proporção nos índices de ferro em crianças que permanecem apenas com o leite materno como fonte alimentar. Em vista disso, se torna necessário a inserção de ferro através de alimentos complementares ricos no mineral [8].

Sendo assim, os dados de estudos indicam que a quantidade apropriada de ferro na alimentação complementar é atingida apenas com a ingestão de alimentos de origem animal em quantidades consideráveis ou através de alimentos enriquecidos com o mineral. A grande dificuldade encontrada é que, em países em desenvolvimento como o Brasil, alimentos ricos em ferro como carnes, peixes e fígado, não são ingeridos em porções suficientes por crianças 
menores de dois anos. Portanto, um método para prover o aporte necessário de ferro à criança é promover o consumo de alimentos fortificados com ferro ou a suplementação medicamentosa do micronutriente [8].

Diante do exposto, a presente pesquisa objetiva conhecer o estado nutricional e a presença de anemia ferropriva em crianças de 3 a 10 anos atendidas em uma Unidade Básica de Saúde no município de Portel/Pará.

\section{Material e métodos}

Tratou-se de um estudo descritivo e transversal, realizado no município de Portel/PA, no período de 30 de novembro a 14 de dezembro de 2012. A amostra foi composta por 170 crianças, na qual foram incluídos aqueles com idade entre 3 a 10 anos de ambos os sexos, que após a leitura do Termo de Consentimento Livre e Esclarecido (TCLE) pelos seus respectivos responsáveis, autorizaram a participação dos menores na pesquisa. Foram excluídos deste estudo os participantes os quais os responsáveis não assinaram o TCLE, que não tiveram condições de realizar a avaliação nutricional ou coleta sanguínea e as crianças que não se enquadravam na faixa etária estipulada.

Esta pesquisa faz parte de um projeto denominado "Marcadores Epidemiológicos em Saúde no Arquipélago do Marajó - Edital FAPESPA023/2009", aprovado no Comitê de Ética do Centro de Hemoterapia e Hematologia do Pará, Fundação HEMOPA, parecer № 0003.0.324.000-10, em 14/07/2010.

A coleta dos dados antropométricos foi realizada na Unidade de Saúde do referido município. Para aferição das medidas antropométricas, utilizou-se balança digital de plataforma da marca EKS 8994, com capacidade de $180 \mathrm{~kg}$ e graduação de peso de 100 gramas, a estatura foi aferida por meio de um estadiômetro portátil WCS com capacidade máxima de $200 \mathrm{~cm}$ de altura e precisão de $1 \mathrm{~mm}$. As crianças foram medidas em decúbito dorsal em superfície lisa e 0 valor registrado em centímetros. Foram usadas como referência as curvas de crescimento da OMS. A classificação das crianças segundo o índice estatura/idade, peso/idade e IMC/idade foi expressa em escore $z$ e adotaram-se os pontos de corte déficit estatural ( $\leq-2$ escores $Z$ ), eutrofia (>-2 escores $z$ ) e magreza ( $\leq-2)$.

Para análise do perfil hematológico das crianças, realizou-se a coleta sanguínea, estando às mesmas em jejum. As amostras foram analisadas in loco, com participação direta da equipe do Laboratório de Análises clínicas (LAC), da Universidade Federal do Pará (UFPA). Para análises hematológicas utilizou-se o aparelho ABX Micros 60 analysers (Horiba-ABX, Montpellier, France), no qual avaliou-se hemoglobina, hematócrito, VCM, HCM e CHCM.

Os valores de referência de normalidade para hemoglobina em crianças de 2 a 6 anos foi de 11,5-13,5 g/dl e de crianças na faixa de 6 a 10 anos de 11,5-15,5 g/dl. O intervalo de normalidade para hematócrito em crianças de 2 a 6 anos foi de 34-40\% e para crianças entre 6 e 10 anos de $35-45 \%$. A referência de VCM para crianças de 2 a 6 anos foi de $75-87 \mathrm{fl}$ e para crianças de 6-10 anos de 77-95 fl. Quanto ao HCM, seus valores normais para crianças de 2 a 6 anos foi de $24-30 \mathrm{pg}$ e para crianças de 6 a 10 anos de 25-33 pg. Em relação ao $\mathrm{CHCM}$, seu valor de referência para crianças de 2 a 6 anos foi de 31-37 g/dl, sendo o mesmo valor padrão para crianças de 6 a 10 anos de idade [9].

Os dados foram analisados no programa Epi info 7, o tratamento estatístico foi realizado no programa BioEstat 5.3 [10], no qual utilizou-se o teste de Kolmogorov-Smirnov, para verificação da normalidade dos dados. O teste Qui-quadrado foi utilizado para comparar as variáveis, correlacionou-se as variáveis antropométricas e hematológicas através do teste de correlação de Pearson. 
Das 170 crianças avaliadas, observou-se que a maior porcentagem (43\%) pertence à faixa etária de 6-10 anos, Gráfico 1.

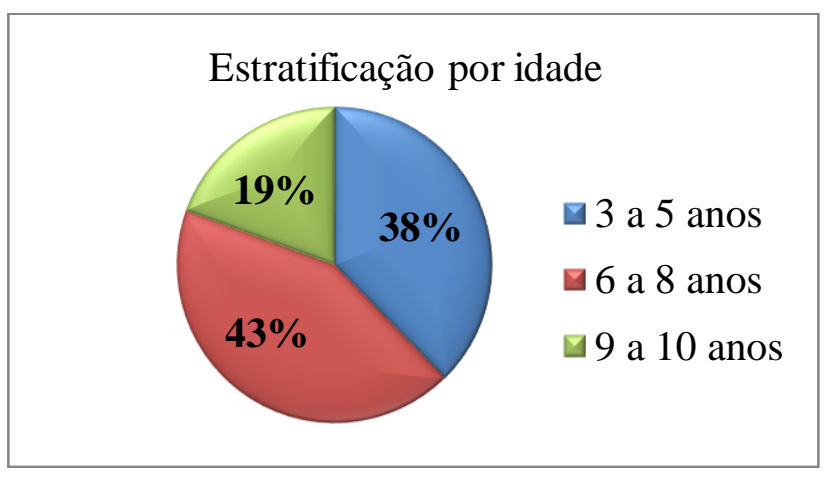

Gráfico 1 - Estratificação por idade de crianças atendidas em uma Unidade Básica de Saúde do município de Portel/Pará.

$\mathrm{Na}$ Tabela I houve predominância do estado de adequação em relação às variáveis pesquisadas, não havendo diferença estatística significativa entre os grupos.

Tabela I - Classificação do estado nutricional através de parâmetros antropométricos de crianças atendidas em uma Unidade Básica de Saúde do município de Portel/Pará.

\begin{tabular}{|c|c|c|c|c|c|c|c|c|}
\hline \multicolumn{2}{|r|}{ Variáveis } & \multicolumn{2}{|c|}{ Todos } & \multicolumn{2}{|c|}{ Meninos } & \multicolumn{2}{|c|}{ Meninas } & \multirow[t]{2}{*}{$p$-valo } \\
\hline & & $\mathrm{N}$ & $\%$ & $\mathrm{~N}$ & $\%$ & $\mathrm{~N}$ & $\%$ & \\
\hline \multirow{3}{*}{$\bar{\alpha}_{*}$} & Baixo & 7 & 4,1 & 3 & 3,0 & 4 & 5,6 & 0.0601 \\
\hline & Adequado & 158 & 92,9 & 91 & 91,9 & 67 & 94,4 & \\
\hline & Elevado & 5 & 2,9 & 5 & 5,1 & & & \\
\hline \multirow{5}{*}{$\sum_{\substack{* \\
*}}^{\bar{U}}$} & Magreza & 6 & 3,5 & 2 & 2,0 & 4 & 5,6 & 0.0779 \\
\hline & Eutrofia & 140 & 82,4 & 81 & 81,8 & 59 & 83,1 & \\
\hline & Risco de sobrepeso & 21 & 12,4 & 13 & 13,1 & 8 & 11,3 & \\
\hline & Sobrepeso & 2 & 1,2 & 2 & 2,0 & & & \\
\hline & Obesidade & 1 & 0,6 & 1 & 1,0 & & & \\
\hline \multirow{3}{*}{$\underset{\substack{\mathbf{W} \\
* \\
* \\
*}}{ }$} & Muito Baixa & 2 & 1,2 & 1 & 1,0 & 1 & 1,4 & 0.0721 \\
\hline & Baixa & 16 & 9,4 & 9 & 9,1 & 7 & 9,9 & \\
\hline & Adequada & 152 & 89,4 & 89 & 89.9 & 63 & 88.7 & \\
\hline
\end{tabular}

*Peso para idade; **Indice de massa corporal para idade; ${ }^{* \star}$ Estatura para idade; Teste estatístico Qui-quadrado.

Tabela II - Parâmetros hematológicos de crianças atendidas em uma Unidade Básica de Saúde do município de Portel/Pará.

\begin{tabular}{|c|c|c|c|c|c|c|c|c|}
\hline & & Todos & & Meninos & & Meninas & & \\
\hline & & $\mathbf{N}$ & $\%$ & $\mathbf{N}$ & $\%$ & $\mathbf{N}$ & $\%$ & $p$-valo \\
\hline & Baixa & 73 & 42,9 & 42 & 42,4 & 31 & 43,7 & 0,0678 \\
\hline \multirow[t]{3}{*}{$\mathrm{Hb}$} & Normal & 94 & 55,3 & 56 & 56,6 & 38 & 53,5 & \\
\hline & Elevada & 3 & 1,8 & 1 & 1,0 & 2 & 2,8 & \\
\hline & Baixa & 32 & 18,8 & 18 & 18,2 & 14 & 19,7 & 0,0559 \\
\hline \multirow[t]{3}{*}{$\mathrm{Ht}$} & Normal & 131 & 77,1 & 77 & 77,8 & 54 & 76,1 & \\
\hline & Elevada & 7 & 4,1 & 4 & 4,0 & 3 & 4,2 & \\
\hline & Baixa & 90 & 52,9 & 50 & 50,5 & 40 & 56,3 & 0,0592 \\
\hline \multirow[t]{3}{*}{ CHCM } & Normal & 80 & 47,1 & 49 & 49,5 & 31 & 43,7 & \\
\hline & Elevada & 0 & 0,0 & & 0,0 & & 0,0 & \\
\hline & Baixa & 153 & 90,0 & 91 & 91,9 & 62 & 87,3 & 0,0518 \\
\hline \multirow[t]{3}{*}{ HCM } & Normal & 16 & 9,4 & 8 & 8,1 & 8 & 11,3 & \\
\hline & Elevada & 1 & 0,6 & & 0,0 & 1 & 1,4 & \\
\hline & Baixa & 131 & 77,1 & 79 & 79,8 & 52 & 73,2 & 0.665 \\
\hline \multirow[t]{2}{*}{ VCM } & Normal & 39 & 22,9 & 20 & 20,2 & 19 & 26,8 & \\
\hline & Elevada & 0 & 0,0 & & 0,0 & & 0,0 & \\
\hline
\end{tabular}

$\mathrm{Hb}=$ hemoglobina; $\mathrm{Ht}$ = hematócrito; $\mathrm{CHCM}$ = concentração de hemoglobina corpuscular média; $\mathrm{HCM}=$ hemoglobina corpuscular média; VCM = volume corpuscular médio; Teste estatístico Qui-quadrado. 
$\mathrm{Na}$ Tabela II encontrou-se $42,9 \%$ das crianças com a hemoglobina abaixo do valor de normalidade, caracterizando algum tipo de anemia. Em relação aos parâmetros hematimétricos ( $\mathrm{CHCM}, \mathrm{HCM}$ e VCM) houve predomínio de valores baixos, em conformidade com os valores de normalidade. Não constatou-se diferença significativa entre os grupos.

$\mathrm{Na}$ Tabela III, quando realizada a correlação de Pearson das variáveis antropométricas com a hemoglobina e o hematócrito, verificou-se que apenas a hemoglobina apresentou uma forte correlação com o IMC/I e moderada para P/I.

Tabela III - Correlação entre parâmetros Antropométricos e Hematológicos de crianças atendidas em uma Unidade Básica de Saúde do município de Portel/Pará.

\begin{tabular}{clccc}
\hline Variáveis & \multicolumn{2}{c}{ Hemoglobina } & \multicolumn{2}{c}{ Hematócrito } \\
\hline $\mathbf{P} / \mathbf{I}$ & $\mathrm{p}=0,032$ & $r=0,632$ & $\mathrm{p}=0,408$ & $r=0,379$ \\
$\mathbf{I M C} / \mathbf{I}$ & $\mathrm{p}=0,002$ & $r=0,881$ & $\mathrm{p}=0,071$ & $r=0,026$ \\
$\mathbf{E} / \mathbf{I}$ & $\mathrm{p}=0,060$ & $r=0,321$ & $\mathrm{p}=0,503$ & $r=0,010$ \\
\hline
\end{tabular}

$\mathrm{P} / \mathrm{I}=$ peso para idade; $\mathrm{IMC} / \mathrm{I}$ = índice de massa corporal para idade; $E / I$ = estatura para idade; $r$ = Coeficiente de Pearson.

Foi identificado que $42,9 \%$ do total de crianças estavam com baixa taxa de hemoglobina, denotando algum tipo de anemia. Deste percentual, foi analisada a quantidade de crianças que apresentaram VCM, HCM e CHCM baixos, e verificou-se que $84,9 \%$ destas crianças apresentaram anemia ferropriva.

Tabela IV - Identificação de anemia ferropriva a partir de índices hematimétricos em crianças atendidas em uma Unidade Básica de Saúde do município de Portel/ PA.

\begin{tabular}{ccccc}
\hline \multicolumn{5}{c}{ Hemoglobina $(\mathbf{H b} \downarrow)-\mathbf{N}=\mathbf{7 3}$ crianças } \\
\cline { 2 - 6 } Índices hematimétricos & $\mathrm{N}$ & $\%$ & \multicolumn{2}{l}{ Outras anemias } \\
& $62 \downarrow$ & 84,9 & $\mathrm{~N}$ & $\%$ \\
VCM $(75-95 \mathrm{fL})$ & $62 \downarrow$ & 84,9 & 11 & 15,1 \\
$\mathrm{HCM}(24-33 \mathrm{pg})$ & $62 \downarrow$ & 84,9 & 11 & 15,1 \\
$\mathrm{CHCM}(31-37 \mathrm{~g} / \mathrm{dL})$ & & 11 & 15,1 \\
\hline
\end{tabular}

$\mathrm{VCM}$ = volume corpuscular médio; $\mathrm{HCM}$ = hemoglobina corpuscular média; $\mathrm{CHCM}$ = concentração de hemoglobina corpuscular média.

Discussão

A faixa etária é um dos aspectos mais importantes a se considerar na anemia ferropriva em crianças, pôde-se perceber que a maior parcela da amostra da pesquisa encontra-se no intervalo dos 3 aos 8 anos de idade. Dentre as doenças nutricionais, a considerada em todo mundo a mais prevalente é a anemia, sobretudo em crianças, porque é nesta fase de vida que ocorre uma velocidade de crescimento aumentada e desenvolvimento acelerado da mesma, e consequentemente ela necessita de um maior aporte de ferro, que se não for suprido pode ocasionar ADF [11].

De acordo com os dados da Tabela I, as crianças que participaram da pesquisa apresentaram-se no geral, em estado de adequação para P/l e E/I e IMC/I. Entretanto, 4,1\% da população estão com baixo $\mathrm{P} / \mathrm{l}$ e $3,5 \%$ em estado de magreza para IMC/I, resultado que se aproxima do encontrado em um estudo realizado por Miglioli et al. [12], que detectou 2,6\% de baixo peso para a idade na população de crianças de municípios de Pernambuco e 1,5\% em estado de magreza para IMC/l. Ainda neste parâmetro, destaca-se que $12,4 \%$ da amostra apresenta risco de sobrepeso, $1,2 \%$ de sobrepeso e $0,6 \%$ de obesidade. O que demonstra que a porcentagem da população que está ganhando peso prevaleceu sobre população em estado de magreza $(3,5 \%)$.

Pesquisas relatam que o fato de a nossa sociedade estar passando por uma transição nutricional, influencia diretamente na modificação dos hábitos alimentares da população, que associado a mudanças socioeconômicas promovem cada vez mais o consumo de produtos extremamente calóricos e pobres em nutrientes como o ferro, necessários para o nosso organismo. E este excesso de peso é prejudicial, ainda mais por ser em crianças, visto que pode desencadear diversos problemas de saúde como hipertensão, diabetes, problemas respiratórios e outros em idade precoce $[11,12]$. 
Em relação aos parâmetros hematológicos (Tabela II e IV), estudos realizados por Nishida et al. [13] em escolas públicas de Maringá/PR, detectaram prevalência da ADF em 39,3\% de sua amostra. Demonstrando que a anemia ferropriva é a mais prevalente dentre as anemias em crianças menores, sendo ainda hoje, um dos maiores problemas de saúde pública no mundo. Estudos reportam que este tipo de anemia resulta da combinação de múltiplos fatores (biológicos, sociais e econômicos) [14]. A dieta representa papel fundamental no desenvolvimento da anemia ferropriva, sendo a causa mais comum à deficiência de ferro dietético segundo a WHO [15].

Segundo Sperandio et al. [16] em seu estudo não foi observado diferença estatística entre os gêneros para anemia, 55\% em meninos e 42,3\% em menina, semelhante aos resultados encontrados da pesquisa.

A Tabela III demonstra que houve uma correlação moderada e significativamente forte entre os valores de hemoglobina com $P / I(p=0,032 ; r=0,632)$ e IMC/I $(p=0,002 ; r=0,881)$, respectivamente. Apesar de peso e IMC se encontrarem adequados na maior parte da população estudada, houve um percentual de crianças $(42,9 \%)$ com baixa hemoglobina no sangue, o que está diretamente relacionado com a ingestão de ferro na alimentação destas crianças. De acordo com Zuffo et al. [17] em seu estudo no município de Capitão/ RS, os hábitos alimentares inadequados, qualidade e quantidade dos alimentos presentes na dieta da criança são componentes associados aos baixos níveis de ferro, e por consequência, redução de hemoglobina produzida no organismo. Oliveira et al. [18] ressaltam que a introdução tardia de alimentos ricos em ferro também contribui para o desenvolvimento da ADF e Menezes et al. [19] referem que esses fatores somados ao rápido crescimento da criança, e portanto rápidas modificações em seu peso e IMC, colaboram para o surgimento da deficiência do mineral e sua evolução para a anemia ferropriva.

Através da Tabela IV, é possível verificar que dentre as anemias encontradas na população de estudo, o maior percentual (84,9\%) recaiu sobre a anemia por deficiência de ferro. Assim como na pesquisa de Silva e Parisi [20] realizada em Tupanciretã/RS, que constatou maior frequência de anemia microcítica e hipocrômica em sua amostra. Este mesmo estudo também corrobora com o fato de os casos de anemia estar distribuídos igualmente entre os sexos.

Estudos referem que a análise do valor de hemoglobina é amplamente utilizada para o diagnóstico de anemia, apesar de não detectar de forma precoce as variações no metabolismo do ferro, uma vez que a anemia ferropriva representa a fase mais avançada da deficiência do mineral no organismo. Mas unindo a observação dos valores de hemoglobina com os de VCM e HCM, por exemplo, temos parâmetros mais sólidos para a identificação da ADF [19].

\section{Conclusão}

Foi encontrada predominância de estado de eutrofia e/ou adequação quanto as variáveis $\mathrm{P} / \mathrm{I}, \mathrm{IMC} / \mathrm{I}$ e E/I. A anemia por deficiência de ferro prevaleceu nas crianças, independente do sexo, em detrimento de outros tipos de anemias.

Estes achados estão relacionados com inúmeros fatores, principalmente com uma dieta que consiste em baixas quantidades do mineral afetando consideravelmente os níveis de hemoglobina no sangue, demostrado pela correlação moderada da hemoglobina com o P/l e forte com IMC/l.

Esses resultados reforçam para o acompanhamento do estado nutricional nos primeiros anos de vida, assim como o planejamento de estratégias de educação alimentar e nutricional para este grupo etário.

\section{Referências}

1. Silva GAP, Costa KAO, Giugliani ERJ. Infant feeding: beyond the nutritional aspects. J Pediatr 2016;3(1):2-7. https://doi.org/10.1016/.jped.2016.02.006

2. Carvalho CA. Consumo alimentar e adequação nutricional em crianças brasileiras: revisão sistemática. Rev Paul Pediatr 2015;33(2):211-21.

https://doi.org/10.1016/j.rpped.2015.03.002

3. Yamagishi JA. Anemia ferropriva: diagnóstico e tratamento. Revista Científica FAEMA 2017;8(1):99-110. https://doi.org/10.31072/rcf.v8i1.438

4. Silva GB, Parisi MM. Results of eritrogram in children with anemia in the municipality of Tupanciretã, RS, Brasil. Revista Brasileira de Análises Clínicas 2017;49(2):141-5. https://doi.org/10.21877/2448-3877.201700558 
5. Novaes TG, Gome AT, Silveira KC, Magalhães EIS, Souza CL, Netto Pereira M et al. Prevalência e fatores associados à anemia em crianças de creches: uma análise hierarquizada. Rev Paul Pediatr 2017;35(3):281-8. https://doi.org/10.1590/19840462/;2017;35;3;00008

6. Penteado BA, Lima WF, Milagres CS. Etiologia de anemia em pacientes renais crônicos em tratamento hemodialítico: Revisão de literatura. Revista Científica da FHO/Uniararas 2017;5(2):18-23.

7. Agência Nacional de Vigilância Sanitária (ANVISA). Anemia por deficiência de ferro. Saúde \& Economia 2013;9:1-5. http://portalarquivos.saude.gov.br/images/pdf/2014/dezembro/15/Anemia-por-Defici-ncia-de-Ferro.pdf

8. Brasil. Ministério da Saúde. Organização Pan Americana da Saúde. Guia alimentar para crianças menores de 2 anos. Brasília MS, 2002. p.32. Disponível em: http://www.redeblh.fiocruz.br/media/guiaaliment.pdf

9. Hoffman R, Benz E, Silberstein L, Heslop H, Weitz J, Anastasi J. Hematology basic principles and practice. 6 ed. USA; 2013.

10. Ayres M. BioEstat 5.3: Aplicações estatísticas nas áreas das ciências biológicas e medicas. Belém/Brasília: Mamiraua/CNPq; 2007.

11. Gontijo TL, Oliveira VC, Lima KCB, Lima PKM. Prática profilática da anemia ferropriva em crianças na estratégia saúde da família. RECOM 2017;7:e1204. https://doi.org/10.19175/recom.v7i0.1204

12. Miglioli TC, Fonseca VM, Junior Gomes SC, Silva KS, Lira PIC, Filho Batista M. Fatores associados ao estado nutricional de crianças menores de cinco anos. Rev Saúde Pública 2015;(49):59. https://doi.org/10.1590/S0034-8910.2015049005441

13. Nishida F.S. Uchimura TT, Szarfarc SC, Bossato TF, Carvalho NA, Uchimura NS. Prevalência de anemia em escolares de escolas públicas de Maringá-PR. Rev Eletr Enferm 2010;12(2):237-44. https://doi.org/10.5216/ree.v12i2.6430

14. Modotti S, Rodrigues JR, Ludwig KM. Comparação do estado nutricional de escolares matriculados na rede pública de ensino da periferia e do centro da cidade de Assis-SP. J Health Sci Inst 2017;35(35):182-6.

15. De Mayer EM, Dallman P, Gurney JM, Hallberg L, Sood SK et al. Preventing and controlling iron deficiency anaemia through primary, health care: a guide for health administrators and programme managers. Geneva. WHO; 1989. 58p. https://apps.who.int/iris/handle/10665/39849

16. Sperandio N, Sant'Ana LFR, Francheschini CC, Priore SE. Comparação do estado nutricional infantil com utilização de diferentes curvas de crescimento. Rev Nutr 2011;24(4):565-74. https://doi.org/10.1590/s1415-52732011000400005

17. Zuffo CRK, Osório MM, Taconeli CA, Schmidt ST, Silva BHC, Almeida CCB. Prevalence and risk factors of anemia in children. J Pediatr 2016;92(4):353-60. https://doi.org/10.1016/j.jpedp.2016.02.008

18. Oliveira CSM, Augusto RA, Muniz PT, Silva AS, Cardoso MA. Anemia e deficiência de micronutrientes em lactentes atendidos em unidades básicas de saúde em Rio Branco, Acre, Brasil. Ciência \& Saúde Coletiva 2016;21(2):517-29. https://doi.org/10.1590/141381232015212.19072014

19. Menezes AEB, Leal LP, Osório MM. Validação de índices hematimétricos para o diagnóstico etiológico da anemia ferropriva em crianças de 6 a 23 meses. Rev Enferm UFPE 2010;4(1):749-56.

20. Silva GB, Parisi MM. Resultados do eritrograma em crianças com anemias do município de Tupanciretã, RS, Brasil. RBAC Revista Brasileira de Análises Clínicas 2017;49(2):141-5. 